\title{
A criterion for regular sequences
}

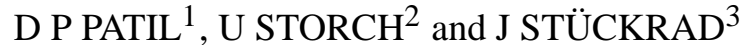 \\ ${ }^{1}$ Department of Mathematics, Indian Institute of Science, Bangalore 560 012, India \\ ${ }^{2}$ Fakultät für Mathematik, Ruhr Universität Bochum, D-44780 Bochum, Germany \\ ${ }^{3}$ Fakultät für Mathematik und Informatik, Universität, Leipzig, D-04109 Leipzig, \\ Germany \\ E-mail: ${ }^{1}$ patil@math.iisc.ernet.in; ${ }^{2}$ uwe.storch@ ruhr-uni-bochum.de; \\ ${ }^{3}$ stueckrad@mathematik.uni-leipzig.de
}

MS received 1 January 2004

\begin{abstract}
Let $R$ be a commutative noetherian ring and $f_{1}, \ldots, f_{r} \in R$. In this article we give (cf. the Theorem in $\S 2$ ) a criterion for $f_{1}, \ldots, f_{r}$ to be regular sequence for a finitely generated module over $R$ which strengthens and generalises a result in [2]. As an immediate consequence we deduce that if $\mathrm{V}\left(g_{1}, \ldots, g_{r}\right) \subseteq \mathrm{V}\left(f_{1}, \ldots, f_{r}\right)$ in Spec $R$ and if $f_{1}, \ldots, f_{r}$ is a regular sequence in $R$, then $g_{1}, \ldots, g_{r}$ is also a regular sequence in $R$.
\end{abstract}

Keywords. Regular sequence.

\section{Regular sequences}

As there is no uniformity about the concept of regular sequence, we first recall the following definitions that we shall use in this note.

\section{DEFINITION 1}

Let $R$ be a commutative noetherian ring and $f_{1}, \ldots, f_{r} \in R$. We say that $f_{1}, \ldots, f_{r}$ is a strongly regular sequence on a $R$-module $M$, if for every $i=1, \ldots, r$ the element $f_{i}$ is a non-zero divisor for $M /\left(f_{1}, \ldots, f_{i-1}\right) M$. The sequence $f_{1}, \ldots, f_{r}$ is called a regular sequence on a $R$-module $M$, if for every $\left.\mathfrak{p} \in \operatorname{Supp}\left(M / f_{1}, \ldots, f_{r}\right) M\right)$, the sequence $f_{1}, \ldots, f_{r}$ in the local ring $R_{\mathfrak{p}}$ is a strongly regular sequence on the $R_{\mathfrak{p}}$-module $M_{\mathfrak{p}}$.

Note that, in contrast to most of the standard text books, we do not assume the $M \neq$ $\left(f_{1}, \ldots, f_{r}\right) M$ for a strongly regular sequence $f_{1}, \ldots, f_{r}$. For general notations in commutative algebra we also refer to [1].

If the sequence $f_{1}, \ldots, f_{r}$ is strongly regular respectively regular on the $R$-module $M$, then the same is true for the sequence $f_{1} \cdot 1_{S}, \ldots, f_{r} \cdot 1_{S}$ on the $S$-module $S \otimes_{R} M$, where $S$ is an arbitrary flat noetherian $R$-algebra.

Note that every sequence is a strongly regular as well as regular sequence on the zero module. Further, it is clear that a strongly regular sequence is a regular sequence but not conversely. For example:

Example. Let $P:=k[X, Y, Z]$ be the polynomial ring in three indeterminates over a field $k, \mathfrak{p}:=P(X-1)+P Z, \mathfrak{q}:=P Y$ and let $R:=P / \mathfrak{p} \cap \mathfrak{q}=P / P Y(X-1)+P Y Z$. Then $Z, X$ is a regular sequence on the $P$-module $R$ but not a strongly regular sequence. 
The difference between regular and strongly regular sequences is well-illustrated in the following statement given in Chapter II, 6.1 of [4].

\section{PROPOSITION}

Let $M$ be a finitely generated module over a noetherian ring $R$ and let $f_{1}, \ldots, f_{r} \in R$. Then the following conditions are equivalent:

(i) $f_{1}, \ldots, f_{r}$ is a strongly regular sequence on $M$.

(ii) For every $s=1, \ldots, r$ the sequence $f_{1}, \ldots, f_{s}$ is a regular sequence on $M$.

It can be easily seen that (see the proof of Proposition 3, Chapter IV, A, $\S 1$ of [5]) a sequence $f_{1}, \ldots, f_{r}$ in a commutative noetherian ring $R$ is a regular sequence for a finitely generated $R$-module $M$ if and only if the Koszul complex $K_{\bullet}\left(f_{1}, \ldots, f_{r} ; M\right)$ gives a resolution of $M /\left(f_{1}, \ldots, f_{r}\right) M$. In particular, if $f_{1}, \ldots, f_{r}$ is a regular sequence on $M$, then for every permutation $\sigma \in \mathfrak{S}_{r}$ the sequence $f_{\sigma 1}, \ldots, f_{\sigma r}$ is also regular for $M$. Further, the above proposition implies that the sequence $f_{\sigma 1}, \ldots, f_{\sigma r}$ is strongly regular on $M$ for every $\sigma \in \mathfrak{S}_{r}$ if and only if all subsequences of $f_{1}, \ldots, f_{r}$ are regular on $M$. For the sake of completeness let us recall Definition 2.

\section{DEFINITION 2}

Let $\left(R, \mathfrak{m}_{R}\right)$ be a noetherian local ring and let $M$ be a non-zero $R$-module. Then the length of a maximal regular sequence on $M$ in the maximal ideal $\mathfrak{m}_{R}$ is called the depth of $M$ over $R$ and is denoted by $\operatorname{depth}_{R}(M)$.

If $M$ is finitely generated then depth can be (cf. [5], Proposition and Definition 3, Chapter IV, A, §2) characterized by

$$
\operatorname{depth}_{R}(M)=\min \left\{i \in \mathbb{N} \mid \operatorname{Ext}_{R}^{i}\left(R / \mathfrak{m}_{R}, M\right) \neq 0\right\} .
$$

A finitely generated $R$-module is called a Cohen-Macaulay module if $\operatorname{dim}_{R}(M)=$ $\operatorname{depth}_{R}(M)$.

\section{Theorem}

The following theorem is the main result of this note.

Theorem. Let $R$ be a commutative noetherian ring, $f_{1}, \ldots, f_{r} \in R$ and let $M$ be a finitely generated $R$-module. Then the following statements are equivalent:

(i) $f_{1}, \ldots, f_{r}$ is a regular sequence on $M$.

(ii) $\operatorname{depth}_{R_{\mathfrak{p}}}\left(M_{\mathfrak{p}}\right) \geq r$ for every $\mathfrak{p} \in \operatorname{Supp}\left(M /\left(f_{1}, \ldots, f_{r}\right) M\right)$.

(iii) $\operatorname{depth}_{R_{\mathfrak{p}}}\left(M_{\mathfrak{p}}\right) \geq r$ for every $\mathfrak{p} \in \operatorname{Ass}\left(M /\left(f_{1}, \ldots, f_{r}\right) M\right)$.

Proof. The implications (i) $\Rightarrow$ (ii) $\Rightarrow$ (iii) are trivial.

(ii) $\Rightarrow$ (i): We may assume that $R$ is local and $f_{1}, \ldots, f_{r} \in \mathfrak{m}_{R}$. Let $\mathfrak{p} \in \operatorname{Ass}(M)$ and let $\mathfrak{q}$ be a minimal prime ideal in $\mathrm{V}\left(\mathfrak{p}+R f_{1}+\cdots+R f_{r}\right)$. Then $\mathfrak{q} \in \operatorname{Supp}\left(M /\left(f_{1}, \ldots, f_{r}\right) M\right)=$ $\operatorname{Supp}(M) \cap \mathrm{V}\left(f_{1}, \ldots, f_{r}\right)$ and so $\operatorname{depth}_{R_{\mathfrak{q}}} M_{\mathfrak{q}} \geq r$ by (ii). Since $\mathfrak{p} \in \operatorname{Ass}(M)$, we have 
$\operatorname{Hom}_{R_{\mathfrak{p}}}\left(k(\mathfrak{p}), M_{\mathfrak{p}}\right) \neq 0$ and so $\operatorname{Ext}_{R_{\mathfrak{q}}}^{h}\left(k(\mathfrak{q}), M_{\mathfrak{q}}\right) \neq 0$ by Chapter 6 , §18, Lemma 4 of [3], where $h:=h t_{R / \mathfrak{p}}(\mathfrak{q} / \mathfrak{p})$. Therefore $r \leq \operatorname{depth}_{R_{\mathfrak{q}}} M_{\mathfrak{q}} \leq h$ (see $(\ddagger)$ in $\left.\S 1\right)$. But then $f_{1} \notin \mathfrak{p}$, since otherwise $h \leq r-1$ by the (generalised) Krull's theorem (see [5], Corollary 4, Chapter III, B, §2). This proves that $f_{1}$ is a non-zero divisor for $M$. Now, induction on $r$ completes the proof.

The implication (iii) $\Rightarrow$ (i) is proved in the lemma which is given below. (In the proof of the lemma we use the implication (ii) $\Rightarrow$ (i).)

\section{COROLLARY 1 ([2], Corollary 1)}

Let $R$ be a commutative noetherian ring, $f_{1}, \ldots, f_{r} \in R$ and let $M$ be a finitely generated $R$-module. Then $f_{1}, \ldots, f_{r}$ is a regular sequence on $M$ if and only if $f_{1}, \ldots, f_{r}$ is a regular sequence on $M_{\mathfrak{p}}$ for every $\left.\mathfrak{p} \in \operatorname{Ass}\left(M / f_{1}, \ldots, f_{r}\right) M\right)$.

\section{COROLLARY 2}

Let $R$ be a commutative noetherian ring and let $f_{1}, \ldots, f_{r}, g_{1}, \ldots, g_{r} \in R$. Let $M$ be a finitely generated $R$-module such that $\operatorname{Supp}\left(M /\left(g_{1}, \ldots, g_{r}\right) M\right) \subseteq \operatorname{Supp}\left(M /\left(f_{1}, \ldots\right.\right.$, $\left.\left.f_{r}\right) M\right)$. Suppose that $f_{1}, \ldots, f_{r}$ is a regular sequence on $M$. Then $g_{1}, \ldots, g_{r}$ is also a regular sequence on $M$. In particular, if $\mathrm{V}\left(g_{1}, \ldots, g_{r}\right) \subseteq \mathrm{V}\left(f_{1}, \ldots, f_{r}\right)$ and if $f_{1}, \ldots, f_{r}$ is a regular sequence in $R$, then $g_{1}, \ldots, g_{r}$ is also a regular sequence in $R$.

From the above equivalence we can also deduce the following well-known fact:

\section{COROLLARY 3 (cf. [5], Theorem 2, Chapter IV, B, §2)}

If $M$ is a finitely generated Cohen-Macaulay module over a noetherian local ring $R$, then every system of parameters of $M$ is a regular sequence on $M$. In particular, in a CohenMacaulay local ring every system of parameters is a regular sequence.

Finally, we give a proof of the lemma which we have already used for the proof of the implication (iii) $\Rightarrow$ (i) of the theorem.

Lemma. Let $R$ be a commutative noetherian ring, $f_{1}, \ldots, f_{r} \in R$ and let $M$ be a finitely generated $R$-module. Suppose that depth $R_{\mathfrak{p}}\left(M_{\mathfrak{p}}\right) \geq r$ for every $\mathfrak{p} \in \operatorname{Ass}\left(M /\left(f_{1}, \ldots\right.\right.$, $\left.f_{r}\right) M$ ). Then $f_{1}, \ldots, f_{r}$ is a regular sequence on $M$.

Proof. We shall prove by induction on $r$ the following implication:

$(*)_{r}:$ If $\operatorname{depth}_{R_{\mathfrak{p}}}\left(M_{\mathfrak{p}}\right) \geq r$ for every $\mathfrak{p} \in \operatorname{Ass}\left(M /\left(f_{1}, \ldots, f_{r}\right) M\right)$, then $f_{1}, \ldots, f_{r}$ is a regular sequence on $M$.

Proof of $(*)_{1}$. Put $f:=f_{1}$ and suppose that $\operatorname{depth}_{R_{\mathfrak{p}}}\left(M_{\mathfrak{p}}\right) \geq 1$ for every $\mathfrak{p} \in \operatorname{Ass}(M / f M)$. Then $\operatorname{Ass}(M) \cap \operatorname{Ass}(M / f M)=\emptyset$. We shall show that $f$ is a non-zero divisor for $M$. Suppose on the contrary that $f$ is a zero divisor on $M$. By localising at a minimal prime ideal in $\operatorname{Ass}(M) \cap \mathrm{V}(R f)$, we may assume that $R$ is a local ring, $\operatorname{depth}_{R}(M)=0$ and that $\operatorname{Ass}(M)=\left\{\mathfrak{p}_{1}, \ldots, \mathfrak{p}_{m}, \mathfrak{m}_{R}\right\}$ with $\mathfrak{p}_{i} \notin \mathrm{V}(R f)$ for all $i=1, \ldots, m$. Then $m \geq 1$. Let $Q_{1}, \ldots, Q_{m}$ and $Q$ be the primary components corresponding to $\mathfrak{p}_{1}, \ldots, \mathfrak{p}_{m}$ and $\mathfrak{m}_{R}$ respectively and let $0=Q_{1} \cap \cdots \cap Q_{m} \cap Q$ be an irredundant primary decomposition of the zero module in $M$. Let $N:=Q_{1} \cap \cdots \cap Q_{m}$. Then $N \neq 0$, Ass $(M / N)=\left\{\mathfrak{p}_{1}, \ldots, \mathfrak{p}_{m}\right\}$ and $f$ is a non-zero divisor for $M / N$, since $f \notin \mathfrak{p}_{i}$ for all $i=1, \ldots, m$. This implies 
that the canonical homomorphism $N / f N \longrightarrow M / f M$ is injective. Further, since $Q$ is $\mathfrak{m}_{R}$-primary in $M$, we have $\mathfrak{m}_{R}^{n} N \subseteq N \cap \mathfrak{m}_{R}^{n} M \subseteq N \cap Q=0$ for some $n \in \mathbb{N}^{+}$, and hence $N$ has finite length. Therefore $N / f N$ has finite length. $\operatorname{But}^{\operatorname{depth}}(M / f M) \geq 1$, since $\mathfrak{m}_{R} \notin \operatorname{Ass}(M / f M)$ and therefore cannot contain any submodules of finite length. This proves that $N / f N=0$ and then $N=0$ by Nakayama's lemma, which contradicts $N \neq 0$.

Proof of $(*)_{r} \Rightarrow(*)_{r+1}$. We may assume that $R$ is local, $f_{1}, \ldots, f_{r+1} \in \mathfrak{m}_{R}$ and $M \neq 0$. Now, we shall prove this implication by induction on $\operatorname{dim}(R)$. Clearly the induction starts at $\operatorname{dim}(R)=0$. Put $\bar{M}_{r}:=M /\left(f_{1}, \ldots, f_{r}\right) M$ and $\bar{M}_{r+1}:=M /\left(f_{1}, \ldots, f_{r+1}\right) M$. Then by induction hypothesis.

$$
f_{1}, \ldots, f_{r+1} \text { is a regular sequence on } M_{\mathfrak{p}} \text { for every } \mathfrak{p} \in \operatorname{Supp}\left(\bar{M}_{r+1}\right) \backslash\left\{\mathfrak{m}_{R}\right\} .
$$

In particular, we have:

$$
\operatorname{depth}_{R_{\mathfrak{p}}}\left(M_{\mathfrak{p}}\right) \geq r+1 \text { for every } \mathfrak{p} \in \operatorname{Supp}\left(\bar{M}_{r+1}\right) \backslash\left\{\mathfrak{m}_{R}\right\} .
$$

We consider two cases:

Case $1 . \mathfrak{m}_{R} \in \operatorname{Ass}\left(\bar{M}_{r+1}\right)$. In this case, by assumption in $(*)_{r+1}, \operatorname{depth}_{R}(M) \geq r+1$. Now, use (ii) $\Rightarrow$ (i) of the theorem to conclude that $f_{1}, \ldots, f_{r+1}$ is a regular sequence on $M$.

Case 2. $\mathfrak{m}_{R} \notin \operatorname{Ass}\left(\bar{M}_{r+1}\right)$. In this case Ass $\left(\bar{M}_{r}\right) \cap \operatorname{Ass}\left(\bar{M}_{r+1}\right)=\emptyset$, $\operatorname{since} \operatorname{depth}{ }_{R_{\mathfrak{p}}}\left(\bar{M}_{r}\right)_{\mathfrak{p}} \geq$ 1 for every $\mathfrak{p} \in \operatorname{Ass}\left(\bar{M}_{r+1}\right) \backslash\left\{\mathfrak{m}_{R}\right\}$ by $(\dagger \dagger)$. Therefore by $(*)_{1}, f_{r+1}$ is a non-zero divisor on $\bar{M}_{r}$. Now, it remains to show that the sequence $f_{1}, \ldots, f_{r}$ is a regular sequence on $M$. For this, let $\mathfrak{p} \in \operatorname{Ass}\left(\bar{M}_{r}\right)$. Since $f_{r+1}$ is a non-zero divisor for $\bar{M}_{r}$, there exists $\mathfrak{q} \in \operatorname{Ass}\left(\bar{M}_{r+1}\right)$ such that $\mathfrak{p} \subseteq \mathfrak{q}$. Note that $\mathfrak{q} \neq \mathfrak{m}_{R}$ and that $f_{1}, \ldots, f_{r}$ is a regular sequence on $M_{\mathfrak{q}}$ by $(\dagger)$ and hence in particular for $M_{\mathfrak{p}}$. This proves that $\operatorname{depth}_{R_{\mathfrak{p}}}\left(M_{\mathfrak{p}}\right) \geq r$ for every $\mathfrak{p} \in \operatorname{Ass}\left(\bar{M}_{r}\right)$ and hence $f_{1}, \ldots, f_{r}$ is a regular sequence on $M$ by $(*)_{r}$.

\section{Acknowledgements}

Part of this work was done while the first author was visiting Germany during AprilJune 2001 under a grant from DAAD, Germany. The first author thanks DAAD, Germany for financial support. The authors sincerely thank Harmut Wiebe for stimulating discussions.

\section{References}

[1] Bruns W and Herzog J, Cohen-Macaulay rings (Cambridge Studies in Advanced Mathematics 39, Cambridge: Cambridge University Press) (1993)

[2] Eisenbud D, Herrmann M and Vogel W, Remarks on regular sequences, Nagoya Math. J. 67 (1977) $177-180$

[3] Matsumura H, Commutative ring theory (Cambridge: Cambridge University Press) (1986)

[4] Scheja G and Storch U, Regular sequences and resultants, Res. Notes in Math. (Natick, Massachusetts: A K Peters) (2001) vol. 8

[5] Serre J-P, Local algebra, in: Springer monographs in mathematics (Berlin, Heidelberg, New York: Springer-Verlag) (2000) 